\title{
Percepções de profissionais sobre o agente comunitário de saúde no cuidado ao idoso dependente
}

\author{
Community health workers caring for dependent elderly people
}

\begin{abstract}
Christina César Praça Brasil (https://orcid.org/0000-0002-7741-5349) ${ }^{1}$
Raimunda Magalhães da Silva (https://orcid.org/0000-0001-5353-7520) ${ }^{1}$

Indara Cavalcante Bezerra (https://orcid.org/0000-0003-0647-2490) ${ }^{2}$

Luiza Jane Eyre de Souza Vieira (https://orcid.org/0000-0002-5220-027X) ${ }^{1}$

Maria do Livramento Fortes Figueiredo (https://orcid.org/0000-0003-4938-2807) ${ }^{3}$

Fernanda Regina Vasconcelos Fernandes Castro (https://orcid.org/0000-0002-5909-9231) ${ }^{1}$

Francisca Francisete de Sousa Nunes Queiroz (https://orcid.org/0000-0002-2933-4974) ${ }^{1}$

Maria Regina Teixeira Ferreira Capelo (https://orcid.org/0000-0003-0423-8676) ${ }^{4}$
\end{abstract}

${ }^{1}$ Programa de Pós-

Graduação em Saúde

Coletiva, Universidade de

Fortaleza. Av. Washington

Soares, 1321, Edson

Queiroz. 60811-905

Fortaleza CE Brasil.

cpraca@unifor.br

${ }^{2}$ Mestrado Profissional em Gestão em Saúde,

Universidade Estadual do

Ceará. Fortaleza CE Brasil.

${ }^{3}$ Programa de Pós-

Graduação em Enfermagem,

Centro de Ciências da

Saúde, Universidade Federal

do Piauí. Teresina PI Brasil.

${ }^{4}$ Centro de Literatura

e Culturas Lusófonas e

Europeias. Funchal Madeira

Portugal.

\begin{abstract}
Community Health Workers (CHWs) are a link between Family Health Strategy (FHS) professionals and the community. This study aims to analyze health care professionals' perceptions and health management about $\mathrm{CHW}$ ' role in caring for dependent elderly people. A qualitative study was carried out, from a hermeneutic-dialectic perspective, with 38 professionals working in elderly people's health in cities in the five regions of Brazil. The results identified CHWs as facilitators of access to elderly people dependent on the health care network, gaps in continuing education and the reduced number of these professionals to meet the demand in primary care. Respondents attributed great importance to CHW's performance, valued their participation in FHS, but pointed out a lack of knowledge and difficulties to supply the adequate amount in health care services and in assistance of elderly people with dependence and family. It is concluded that health professionals and managers consider CHWs to be a great workforce and essential to the dependent elderly people and their caregivers. The number of CHWs is insufficient in relation to FHS, and there is a lack of training to deal with various types of dependence. Key words Community health worker, Family health strategy, Health personnel, Frail elderly, Qualitative research
\end{abstract}

Resumo O Agente Comunitário de Saúde (ACS) é um elo entre os profissionais da Estratégia Saúde da Família (ESF) e a comunidade. Objetivou-se analisar as percepçães de profissionais da atenção e da gestão em saúde sobre as atribuições do ACS no cuidado ao idoso dependente. Realizou-se um estudo qualitativo, na perspectiva hermenêuticadialética, com 38 profissionais que atuavam na saúde do idoso em municípios das cinco regiões do Brasil. Os resultados identificaram o ACS como facilitador do acesso do idoso dependente à rede de atenção em saúde, lacunas na Educação Permanente e um número reduzido desses profissionais para atender a demanda na atenção primária. Os respondentes atribuíram grande importância à atuação do ACS, valorizaram a sua participação na Estratégia Saúde da Família, mas apontaram a deficiência de conhecimento e dificuldades para suprir a quantidade adequada nos serviços de atenção à saúde e na assistência do idoso com dependência e familiares. Conclui-se que os profissionais e gestores da saúde consideram os ACS uma grande força de trabalho e com atuação imprescindivel junto ao idoso dependente e seus cuidadores. O número de ACS é insuficiente em relação à ESF e falta capacitação para lidar com os variados tipos de dependência.

Palavras-chave Agente comunitário de saúde, Estratégia saúde da família, Profissionais de saúde, Idoso dependente, Pesquisa qualitativa 


\section{Introdução}

Os agentes comunitários de saúde (ACS) são trabalhadores do Sistema Único de Saúde (SUS) do Brasil que iniciaram as atividades no Programa Nacional de Agentes Comunitários de Saúde (PNACS), o qual, após um ano, passou a ser denominado Programa de Agentes Comunitários de Saúde (PACS) ${ }^{1}$. A profissão foi regulamentada em 2002 e suas atribuições estão previstas na Portaria no 2.4361 de setembro de 2017, que aprova a nova Política Nacional de Atenção Básica - PNAB $^{1,2}$.

O Programa de Agentes Comunitários de Saúde iniciou no final dos anos 1980, inspirado em experiências exitosas de alguns estados do Nordeste brasileiro, Distrito Federal e São Paulo ${ }^{3}$. Pessoas de comunidades carentes, especialmente mulheres, foram designadas para auxiliar na promoção da saúde nos respectivos territórios. O ACS fortaleceu a ligação da comunidade com os serviços de saúde e deu suporte a populações menos favorecidas, a exemplo do que acontece em programas similares de outros países ${ }^{4,5}$.

O Programa Saúde da Família (PSF), posteriormente Estratégia Saúde da Família (ESF), passou a agregar funções do ACS tanto nas Unidades Básicas de Saúde (UBS) quanto nas Equipes de Saúde da Família (EqSF). Dentre as suas atribuições, destaca-se exercer o elo entre as demandas da comunidade e os serviços de saúde localizados em sua área de adscrição ${ }^{6,7}$.

Em sistemas de saúde de vários países, há trabalhadores cuja função se assemelha a dos ACS brasileiros. Apesar das diferentes denominações, o termo Community Health Workers os identifica na literatura internacional ${ }^{4,5,8}$. Independentemente dos países de origem, esses profissionais revelam traços comuns, desempenhando papel estratégico junto a populações de áreas rurais, afastadas ou em situação de vulnerabilidade. Sua importância é reconhecida na interlocução com a comunidade, fortalecimento e consolidação de programas de saúde em diferentes países $^{4,8}$.

Nos países da América do Sul, exceto o Uruguai, os profissionais que se assemelham ao ACS brasileiro são integrantes oficiais das equipes multiprofissionais, atuando com remuneração ou voluntariamente ${ }^{5}$. Portugal, França, Espanha, Inglaterra, Estados Unidos, Canadá, Índia, Kenya, Uganda, Ghana e África do Sul também contemplam esses profissionais ${ }^{4,8,9}$, que atuam junto a uma quantidade pré-estabelecida de famílias, em uma base geográfica definida ${ }^{10}$.
No Brasil, em 2017, a nova $\mathrm{PNAB}^{2}$ suprimiu o número mínimo de ACS por equipe, contrapondo-se à normatização anterior de quatro ACS por equipe, podendo haver até um ACS por equipe. Acrescenta-se que também excluiu do texto a previsão da carga horária de até oito horas/semanais destinadas à formação desses profissionais ${ }^{2}$.

De acordo com os princípios da educação e promoção da saúde, o ACS é o profissional que convive com as singularidades das famílias, conhecendo as condições de vida e as relações sociais nas comunidades ${ }^{11,12}$. Dentre as suas funções, participar do mapeamento do território, do cadastramento das famílias e da visita domiciliar permite o conhecimento da realidade comunitária e a identificação de populações de risco ${ }^{13}$.

Com o aumento da expectativa de vida e o envelhecimento da população, surgiram desafios assistenciais e sociais dirigidos aos ACS, pois as mudanças demográficas, epidemiológicas e sanitárias resultaram na expansão de doenças crônicas e complexas, que tornam as pessoas idosas cada vez mais frágeis e dependentes, demandando redes de atenção à saúde (RAS) mais organizadas e resolutivas ${ }^{12}$.

Com efeito, o aumento da longevidade requer o envolvimento de toda a EqSF no cuidado ao idoso. Nesse sentido, o contato longitudinal e a proximidade do ACS aos domicílios expressam um espaço potente para a realização do cuidado, especialmente ao idoso dependente ${ }^{14}$.

Acredita-se que as diretrizes vigentes na PNAB que reduziram o número de ACS por equipe podem comprometer a cobertura assistencial das famílias, além de fragilizar as ações de educação permanente e treinamento dos ACS. Isto dificulta o atendimento das necessidades da população, incluindo o idoso dependente, o qual requer atenção articulada e resolutiva. Nesse contexto, o ACS exerce papel estratégico, diante da sua proximidade com as famílias no território. No entanto, evidencia-se uma lacuna na produção científica sobre a relevante atuação do ACS junto ao idoso dependente, o que justifica focalizar, nesta investigação, o olhar de profissionais e gestores de saúde sobre as ações e os papéis dos ACS junto à população idosa frágil.

Ante o exposto, o artigo objetiva analisar as percepções de profissionais da atenção básica e da gestão de saúde sobre a atuação do ACS no cuidado ao idoso dependente. 


\section{Método}

Realizou-se um estudo qualitativo, com apoio na hermenêutica-dialética, uma vez que buscou a compreensão do problema, levando-se em consideração aspectos objetivos, subjetivos, crenças e significações dos gestores e profissionais da saúde em relação à atuação do ACS no contexto do cuidado ao idoso dependente e suas famílias.

A hermenêutica-dialética, como lupa teóricometodológica, possibilita estabelecer um processo reflexivo ${ }^{15}$ para compreender a comunicação entre as pessoas e suas conexões com o ambiente. Nessa perspectiva, a vida cotidiana e o senso comum são elementos de investigação, sendo as linguagens que permeiam as vivências e as interações instrumentos que levam ao entendimento das características do universo sob investigação ${ }^{16}$. O pesquisador desnuda-se das concepções pessoais, o que possibilita a leitura dos sentidos que emergem do movimento reflexivo.

Ao associar a hermenêutica e a dialética ${ }^{17}$, consensos e dissensos levam a uma visão mais ampla de mundo, possibilitando que a pesquisa se aprofunde no contexto e revele as múltiplas fases de uma realidade complexa e permeada por valores, crenças e subjetividades ${ }^{18,19}$. Essa junção de métodos sintetiza os processos compreensivos e críticos, pois a mesma razão que compreende, esclarece e reúne, também contesta, dissocia e critica ${ }^{15}$.

As contribuições dessa abordagem na compreensão das particularidades e dos conceitos atribuídos à atuação dos ACS junto ao idoso pelos respondentes foi verificada com suporte em experiências, conflitos, vivências e interlocuções mantidas no âmbito profissional e comunitário.

A coleta de dados aconteceu de junho a setembro de 2019, em oito municípios das cinco regiões do Brasil: Araranguá, Brasília, Fortaleza, Manaus, Porto Alegre, Belo Horizonte, Rio de Janeiro e Teresina.

Participaram 38 profissionais da atenção primária e da gestão em saúde, sendo 22 do sexo feminino e 16 do masculino, com média de idade de 43 anos, que atuavam na saúde do idoso nos oito municípios, com as seguintes formações: médicos (8), enfermeiros (18), assistentes sociais (3), educador físico (1), fisioterapeuta (1), dentistas (2), neuropsicólogo (1), biólogo (1), técnico de Enfermagem (1), Pedagogo (1) e ACS (1).

Seguindo os critérios de inclusão da pesquisa, foram convidados profissionais da saúde atuantes, há pelo menos um ano, em UBS e em programas municipais que oferecem suporte a idosos e suas famílias. Como gestores, foram convidados aqueles responsáveis pelos órgãos que coordenam a saúde do idoso nos referidos municípios, a exemplo de secretários de saúde ou gestores de programas específicos. De modo geral, os participantes do estudo tinham especializações, experiências prévias ou cursos de educação continuada que os habilitavam a atuar no contexto da saúde do idoso. Foram excluídos do estudo aqueles que estavam de férias, afastados do serviço ou que faltaram ao trabalho no dia da coleta.

Para a composição da amostra, identificaram-se os profissionais e gestores nas UBS e nas secretarias municipais de saúde das respectivas cidades, os quais foram convidados, presencialmente ou por contato telefônico, a participar das entrevistas. Estas foram conduzidas nos locais de trabalho dos participantes por pesquisadores experientes. Portanto, a amostra foi realizada intencionalmente, o que é demonstrado por municípios, na Tabela 1.

As entrevistas semiestruturadas foram direcionadas a cada tipo de profissional, com questionamentos que iniciaram abordando dados de identificação e tempo de atuação no serviço e foram se aprofundando para capturar a percepção dos entrevistados sobre o idoso com dependência social, física, mental e cognitiva, facilidades e dificuldades para acolher, acompanhar ou encaminhar os idosos e seus familiares com os diversos tipos de dependência, e iniciativas para apoiar o idoso e as famílias.

As perguntas das entrevistas foram elaboradas pelos pesquisadores que atuaram conjuntamente na investigação, os quais participaram das equipes locais, realizaram as entrevistas com permissão da gravação em áudio e foram transcritas na íntegra. As anotações registradas em diário de campo também subsidiaram a coleta de dados, que se encerrou a partir do princípio da saturação ${ }^{20}$, quando não mais emergiram novas ideias relacionadas ao tema em debate.

Em consonância com os pressupostos da hermenêutica-dialética, seguiram-se as três etapas recomendadas por Minayo $^{17}$ - ordenação, classificação e análise final dos dados. A ordenação abrange a transcrição de entrevistas, leitura do material empírico e organização dos conteúdos que emergem do texto em conjuntos de sentidos. Classificar os indicadores denota a elaboração e o agrupamento dos conjuntos de sentidos em temas específicos do objeto de estudo. A análise consistiu na elaboração de um fluxograma de organização para a visualização das temáticas.

Embora o roteiro de entrevista não incluísse questões específicas sobre o papel do ACS no 
Tabela 1. Distribuição do número dos participantes, por município, Brasil, 2020.

\begin{tabular}{lcc}
\hline \multicolumn{1}{c}{ Municípios } & Profissionais & Gestores \\
\hline Araranguá - SC & 5 & 4 \\
Belo Horizonte - MG & 2 & 4 \\
Brasília - DF & 2 & 1 \\
Fortaleza - CE & 2 & 2 \\
Manaus - AM & 2 & 2 \\
Porto Alegre - RS & 2 & 1 \\
Rio de Janeiro - RJ & 5 & 1 \\
Teresina - PI & 2 & 1 \\
Total & 22 & 16 \\
\hline
\end{tabular}

Fonte: Autoria própria (2020).

contexto da saúde do idoso dependente, o foco de análise deste artigo recaiu sobre as 14 falas de gestores e 18 de profissionais da saúde que versam sobre este assunto. Assim, diante da frequência e da relevância, os dados fizeram emergir o objetivo do presente estudo. O material, após organizado e analisado, foi apresentado em duas temáticas.

Os dados foram interpretados à luz da hermenêutica-dialética ${ }^{15,17}$, da literatura que versa sobre a atuação do ACS e das abordagens relacionadas à saúde do idoso ${ }^{2,3,21}$. Ao final, a equipe de pesquisa sintetizou as descobertas, dialogando com os temas identificados e comparando-os ao referencial teórico-metodológico, aos objetivos e aos pressupostos previamente estabelecidos.

Para proteger as identidades dos participantes, optou-se por denominá-los por meio letras e números. As letras PS remetem a profissional da saúde e a letra $\mathrm{G}$ diz respeito a gestor. Os números correspondem à quantidade de participantes, como, por exemplo, PS1 significa profissional da saúde número 1, G1 é gestor 1 e, assim, sucessivamente.

Este artigo consiste em um recorte do Estudo situacional dos idosos dependentes que residem com suas famílias visando a subsidiar uma política de atenção e de apoio aos cuidadores, coordenado por uma equipe pertencente ao Departamento de Estudos sobre Violência e Saúde Jorge Careli, da Escola Nacional de Saúde Pública, Fundação Oswaldo Cruz. O projeto foi aprovado pelo Comitê de Ética e Pesquisa da Fundação Oswaldo Cruz.

\section{Resultados e discussão}

As percepções dos profissionais da saúde, sejam eles da atenção ou da gestão, sobre a atuação dos ACS, expressas nas entrevistas, foram mapeadas e sintetizadas, convergindo para a formulação de duas categorias temáticas: "ACS como facilitador do acesso do idoso dependente à rede de atenção em saúde" e "Lacunas na educação permanente dos ACS sobre a assistência ao idoso dependente".

\section{ACS como facilitador do acesso do idoso dependente à rede de atenção em saúde}

A aproximação do ACS com a comunidade em que atua e reside facilita o conhecimento desse profissional quanto aos problemas sociais e de saúde das famílias. Nessa lógica, a práxis do ACS se dá na capilaridade do primeiro nível de atenção à saúde, materializando-se no fácil acesso ao idoso e à sua família, no envolvimento com a dinâmica familiar dos idosos, estabelecendo a formação de vínculos, a 'ponte'. Essa dinâmica avança para além da permeabilidade junto aos moradores, pois decorre, também, do conhecimento dos códigos éticos e de segurança que regem o território de atuação $0^{22}$.

[...] É o trabalho do ACS faz muito bem esse papel [...], de ponte. (PS 1, Rio de Janeiro)

$O$ agente de saúde é fundamental porque ele está dentro do território, ele sabe onde estão todos os idosos. A gente [profissionais de saúde] cadastrou esses idosos, fez essa estratificação [...], então ele sabe quem são essas pessoas, ele sabe a realidade de cada família e onde eles estão [...]. (G1, Fortaleza)

[...] O agente de saúde sempre vai acompanhando para não ir sozinha [...], já fiz sozinha [...]. Por uma questão de segurança da área a gente não faz sozinho [...]. (PS 3, Porto Alegre)

Esta temática atesta que o ACS é visto como uma ponte entre os profissionais da saúde, o serviço e a comunidade assistida. Essa ideia de ponte, referida com recorrência pelos participantes, remete à ligação, elo e vínculo ${ }^{23}$. Esta percepção transcorre com base na reorientação do modelo de atenção à saúde que assume o entendimento de território como espaço vivo, onde pessoas demonstram singularidades, modos de conviver e arranjos socioculturais para enfrentar os adoecimentos ${ }^{6}$.

Nas falas dos participantes emerge a importância da atenção domiciliar realizada pela equipe de saúde junto ao ACS, firmando-se como ponto de atenção na RAS e corroborando a con- 
cepção da visita domiciliar (VD) como meio para efetivar o cuidado centrado nas singularidades e nos contextos de vida ${ }^{24}$. Vale exprimir que a realização da VD fortalece o sentimento de pertença dos idosos aos serviços de saúde.

$O$ agente de saúde indica quem está precisando mais, e a gente seleciona qual idoso está precisando ser visitado. E, no máximo, fazemos seis visitas pela manhã. A gente faz um rodizio de visitas [...] (PS 7, Teresina)

[...] Na visita que os agentes de saúde fazem, conversam e tomam chá. Isso não é perder tempo. Isso é dar atenção para aquele idoso, ele se sente importante [...]. O agente de saúde está vindo na minha casa, a enfermeira está vindo na minha casa para me ver [...]. Eu acho que é importante, os idosos se sentem valorizados [...] (PS 6, Porto Alegre)

Importante é sinalizar a integração ensinoserviço, reiterando o SUS como "cenário de práticas” imprescindível à formação, destacando a mediação do ACS nos percursos acadêmicos e na constituição da intersetorialidade, com acesso do idoso dependente às ações multiprofissionais dos serviços de saúde ${ }^{25}$.

[...] Temos dois estudantes estagiários no acolhimento e mais um agente de saúde que destina para o profissional que está de plantão. Então, se tiver um problema grave e a família vir, vai ser atendido no mesmo dia. (PS 7, Teresina)

[...] Aqui nós temos muitos estudantes, então eles [ACS] levam os estudantes da fisioterapia, levam os alunos da farmácia, [...] levam profissionais para conversar com esses idosos e acaba se tornando um grupo cooperativo. [...] Eles conversam, tiram dúvidas [...]. (G 4, Fortaleza)

$\mathrm{Na}$ perspectiva de aproximação com os usuários do sistema de saúde, o ACS atua como articulador na ESF. A maioria deles, oriunda das comunidades, denota características socioculturais que os achegam aos usuários, além de permitir contextualizar os problemas no território adscrito $^{26}$.

Ainda no sentido de aproximação com a comunidade, o ACS realiza o acolhimento, a escuta e o acompanhamento dos idosos nos domicílios, oferecendo maior conforto e buscando a resolubilidade das demandas ${ }^{27}$.

Litzelman et al. $^{28}$ reportam resultados na redução dos atendimentos de urgência e nas hospitalizações no Estado de Indiana, nos Estados Unidos da América, onde o idoso participa das discussões e decisões sobre seus cuidados com os agentes de saúde locais, no domicílio, por meio do planejamento de cuidados. Isso mostra a importância da aproximação desse profissional com os usuários do sistema de saúde. O estudo ressalta a necessidade de uma estratégia para aumentar a quantidade de países com esse mesmo direcionamento, conforme aponta a Organização Mundial da Saúde, ao estabelecer recomendações programáticas e financeiras para os programas que incluem a figura dos agentes de saúde ${ }^{29}$.

A exemplo da Índia, os agentes de saúde estabelecem uma ligação entre os líderes da comunidade e os das organizações religiosas para o desempenho de cuidados de saúde, favorecendo o acesso das comunidades carentes e remotas em todo o país à atenção básica. Nessa perspectiva, essa atuação proporciona mudanças de comportamento relacionados à saúde, repercutindo na expansão da assistência à saúde dessas populações, bem como o impacto positivo na saúde em toda a Índia ${ }^{30}$.

Pesquisadores australianos observaram, a partir dos exemplos de programas governamentais bem-sucedidos em países de baixa e média renda, que os Trabalhadores da Saúde Comunitária são eficazes. Assim, decidiram avaliar o escopo, o valor desse tipo de programa na Austrália e os desafios na integração desses trabalhadores no sistema de saúde. Verificaram a relevância da sua atuação na melhoria da equidade e do acesso da população menos favorecida à Atenção Primária à Saúde (APS), facilitando o acesso aos serviços e informações de saúde, o que se torna ainda mais importante diante do número crescente de doenças crônicas ${ }^{31}$.

Sob a óptica dos profissionais da atenção e gestão, a atuação dos ACS oferece melhoria das condições de saúde e avanços das ações preconizadas na PNAB. Assim, a interlocução desses trabalhadores com a comunidade é vista como um aspecto positivo nas condições relacionadas à comunicação e à educação em saúde.

No âmbito dessa aproximação, o papel do ACS remete a aspectos importantes no contexto da saúde do idoso dependente, uma vez que amplia a captação dessa população para acessar cuidados primários (busca ativa), possibilita a estratificação de risco, a identificação de doenças e aproxima as demandas dos idosos às $\mathrm{UBS}^{32}$.

Nem toda "captação" das necessidades de saúde do idoso dependente contém desfecho resolutivo; muitas vezes, pode se tornar reprimida em função dos processos organizativos do SUS, a exemplo da regulação e dos percalços para transpor os níveis de complexidade da atenção à saú$\mathrm{de}^{27}$.

É uma demanda que muitas vezes fica adormecida no território [...] O Agente de Saúde sabe dis- 
so, mas nem sempre tem instrumentos para cuidar dessa situação. (G1, Fortaleza)

Em contraposição, existem dificuldades com a redução do número de ACS por equipe ${ }^{2}$, o que implica a fragmentação e a incompletude do cuidado ao idoso dependente, família e ou cuidador $^{33}$. Fatos desta natureza foram identificados nas falas dos profissionais de Araranguá e Belo Horizonte:

[...] A nossa atenção básica tem pouca perna também para chegar ao idoso, o que seria ideal. Eu gostava muito quando a gente tinha mais ACS [...]. Nessa situação, estamos com um agente por equipe, não tem como [...]. (G2, Araranguá)

[...] Agora, nós estamos com três ACS. A nossa área é enorme, são 40 mil pessoas [risos] [...]. Então, não tem condição dos ACS visitarem todas as famílias [...]. (P1, Belo Horizonte)

A redução do número de agentes por equipe entrou em vigor com a nova PNAB, em 2017. Esta determinação legal fragiliza a cobertura assistencial cotidiana dos ACS, em seus territórios, comprometendo o cumprimento da agenda de visitas domiciliares, momento no qual são levantados os problemas de saúde e as necessidades dos idosos dependentes. A partir de então, os ACS agendam consultas e atendimentos dos profissionais de suas equipes, os quais, por sua vez, fazem os encaminhamentos e as referências aos níveis especializados de saúde.

Depreende-se que as relações de proximidade e de interlocução mantidas pelos ACS e os profissionais da atenção e gestão, na tentativa de mediar e aproximar idosos e familiares dos serviços de saúde, intentam oferecer maior resolubilidade para aspectos que, muitas vezes, ficam não revelados dentro dos lares e famílias dos idosos dependentes.

Muitos cuidadores, por serem sozinhos e não contarem com apoio de outros familiares ou vizinhos, veem nos ACS um suporte que viabiliza a busca por atendimento, a obtenção de medicamento ou, simplesmente, um ouvido amigo para aliviar o sofrimento e as limitações do idoso ou deles próprios.

A criação do "El libro Blanco de la Dependência”, na Espanha, em 2004, exemplifica o amplo cuidado ao idoso dependente, considerando a integralidade da saúde dessa população no contexto da rede de cuidados. Assim, estabelece vários eixos de ação e destaca o apoio ao idoso e ao cuidador no domicílio com uma forma de respeito, proteção, conforto e melhoria da qualidade de vida e saúde ${ }^{34,35}$. O PACS pode buscar nessa estratégia inspiração para que atue na dire- ção de um cuidar mais integral dessas famílias, a partir do amparo das políticas públicas e de uma formação dos ACS direcionada à saúde do idoso.

\section{Lacunas na educação permanente dos ACS sobre a assistência ao idoso dependente}

Identificou-se, nas falas dos profissionais, o entendimento sobre o desejo de contar, em suas equipes, com ACS qualificados, detentores de conhecimento e destreza para utilizarem meios em educação em saúde, favorecendo a sua atuação junto ao idoso dependente.

O objetivo [...] seria possibilitar uma atuação mais adequada do ACS durante a visita domiciliar de um idoso dependente, tanto no sentido de atenção às condições que possam comprometer mais a saúde do idoso como a do cuidador. Então, com informação mais adequada, com identificação de situações [...] o ACS pode colaborar e melhorar a atuação da equipe no domicílio. (G2, Fortaleza)

Estudos $^{22,36}$ afirmam que as práticas do ACS estão pautadas no acolhimento e na escuta qualificada, o que fortalece a relação com os idosos e, relembram, que a atuação do ACS no cuidado à população idosa advém das orientações da Política Nacional de Saúde da Pessoa Idosa. Dentre outras diretrizes, esta política valoriza a formação e a educação dos profissionais do SUS para atuar junto a essa população, o que traz valorização ao trabalho do ACS e contribui para a capacitação desses profissionais na perspectiva do envelhecimento.

Silva et al. ${ }^{37}$ e Medeiros et al. ${ }^{38}$ apontam que os desafios que cercam o cuidado ao idoso dependente e as iniquidades sociais em saúde ampliam as situações de vulnerabilidade. Nesse sentido, os participantes reúnem esforços para amenizar esta dependência e cuidados negligenciados, promovendo ações de saúde em consonância com os propósitos do envelhecimento ativo.

[...] A gente fez uma amostra das iniciativas que nós temos na unidade. E uma das coisas que os próprios ACS estão fazendo é um grupo que funciona [...] numa praça aqui próximo. Nós tentamos fazer um grupo de idosos dentro da unidade, eles não vinham [...]. E, aí, eles começaram a fazer na praça e começou a juntar os idosos. (G 1, Fortaleza)

Rahmawate e Bajorek ${ }^{39}$ exploram o papel do agente de saúde em um programa de apoio a idosos hipertensos moradores de uma comunidade rural na Indonésia. Esses profissionais destacaram-se como "guardiões da assistência médica”, visto que, após capacitados, auxiliaram no monitoramento da hipertensão, apoiaram 
os profissionais de uma unidade de saúde na aferição da pressão arterial, orientações sobre atividade física e alimentação saudável. Idosos e profissionais de saúde evidenciaram muitos benefícios à saúde dos assistidos a partir da atuação dos agentes de saúde, além do sentimento de amparo e apoio dos pares.

Existe alinhamento das ações dos ACS brasileiros descritas pelos profissionais e gestores com os princípios da integralidade e intersetorialida$\mathrm{de}^{40}$, priorizando a dependência do idoso, o que corrobora com a fala dos entrevistados:

[...] a gente tem algumas pautas fixas. Entre elas, tem os pacientes acamados, que a maioria é idoso. E também os ACS trazem os casos para a gente, e a gente também leva para os ACS para fazer a busca ativa de um idoso, para saber se algum idoso está em situação de vulnerabilidade social [...] (PS 2, Belo Horizonte)

[...] Nas visitas domiciliares, quando a gente vai fazer com os ACS, a gente tenta orientar bastante a família [...] sobre o paciente acamado e sobre o paciente que não tem essa restrição [...] (PS 1, Porto Alegre)

É crucial a educação permanente em saúde para que se possa ofertar ações de qualidade. Esta deve ser fundamentada no conhecimento das diretrizes políticas, estatutos e manuais ${ }^{41,42}$, além de dispositivos legais e éticos. Melo et al..$^{43}$ afirmam que, ao entender o SUS como espaço de formação, a PNAB 2011 previa que até $8 \mathrm{~h} /$ semanais dos profissionais das EqSF fossem utilizadas para atividades de formação, educação permanente, apoio matricial e plantão na rede de urgência. Não perdurou na PNAB vigente esta redação ${ }^{1,2}$.

A reformulação da PNAB fragiliza a educação permanente no propósito maior, que é a reconfiguração da práxis em busca de soluções para os problemas que dela emanam. Desse modo, percebe-se, também, como descontinuidade, uma menor valorização da educação permanente, peçachave para o trabalho na Atenção Básica $(\mathrm{AB})^{42}$. A fala de um gestor evidencia o quão importante é internalizar as concepções da educação permanente no trabalho em saúde:

[...] Foram treinados vários profissionais e ACS em práticas integrativas [...] e eles ficaram adormecidos na rede. Fizeram o curso e ficaram parados, não estavam fazendo as suas práticas [...] (G 2, Fortaleza)

Filgueiras e Silva ${ }^{26}$ assinalam que esses profissionais facilitam a aproximação entre o serviço de saúde e a comunidade, bem como auxiliaram na ampliação do acesso às informações e práticas de saúde. Estudos ${ }^{4,44}$ demonstram que o trabalho dos ACS pode ser eficaz para melhorar a saúde da população em países de baixa, média e alta renda. No entanto, os ACS não podem atingir todo o seu potencial de atuação na comunidade, com diferentes populações e faixas etárias, sem um processo de formação continuada.

A compreensão da importância da atuação do ACS pelos gestores e profissionais da saúde, na perspectiva da dialética, advém do ato comparativo, quando confrontam as ações e as atribuições de cada um. Nesse sentido, os próprios ACS reconhecem a necessidade de uma formação mais ampla e profunda que privilegie a saúde do idoso e a dependência, para que possam atuar com maior propriedade e ofereçam mais resolubilidade a essa população.

Nesta conformação lógica, as Diretrizes para Capacitação dos ACS em Linhas de Cuidado ${ }^{21}$ baseiam-se em eixos para a realização de um cuidar mais direcionado ao público-alvo, propondo atender as demandas regionais, locais e as prioridades de saúde. Elegem pontos imperativos para que se efetivem, na APS, sendo este o nível que ordena e coordena o cuidado nos meandros das RAS.

A capacitação do ACS em saúde do idoso é vista pelos participantes como estratégia essencial para que este profissional possa atuar fundamentado pelas políticas de saúde do Brasil que regulamentam a organização dos serviços de saúde e a melhoria das condições de vida e saúde da população. De tal maneira, poderá atuar na promoção e no cuidado à saúde do idoso, por meio da articulação entre ações de educação e mediação em saúde, princípios da educação popular, problematização da realidade e participação ativa na comunidade. Com efeito, o ACS poderá estimular cada vez mais as relações emancipatórias entre os distintos profissionais da saúde atuantes junto à população idosa, desenvolvendo ações integradas nas equipes da atenção básica e fortalecendo o princípio da coordenação do cuidado.

\section{Considerações finais}

Os achados revelam a visão positiva e a relevância do papel dos ACS nas equipes de saúde, especialmente, nas da ESF. As ações de saúde destinadas aos idosos, sobretudo, os dependentes, necessitam do desempenho e da atuação dos ACS, pois estes profissionais representam um elo entre a família, a EqSF e outros serviços da rede de saúde dos municípios.

As falas dos participantes demonstram que graças à intensiva aderência dos ACS junto às 
famílias cadastradas nas suas áreas adstritas, as EqSF têm uma visão permanente para esta clientela; em especial a população idosa, frágil e dependente.

Por ter maior proximidade com as famílias, os ACS identificam as necessidades dos idosos dependentes e facilitam seu acesso à rede de atenção à saúde. Os profissionais reconhecem, no entanto, que a redução do número de ACS por equipe, bem como o comprometimento da carga horária destinada à capacitação e educação permanente desses agentes, respondem pela fragilidade da atuação e da cobertura assistencial. Isto compromete toda a linha de cuidado aos idosos dependentes, seja na atenção primária ou no acesso aos outros níveis de assistência à saúde.

Outra evidência relatada pelos participantes sobre o desempenho dos ACS nos seus territórios refere-se ao apoio prestado aos cuidadores informais, uma vez que reconhecem nele um suporte que viabiliza a busca por atendimento, a obtenção de medicamento ou uma escuta atentiva capaz de aliviar o sofrimento e as limitações do idoso e dos próprios cuidadores.

Ainda a respeito da atuação dos agentes junto aos idosos frágeis, os participantes apontaram a necessidade de mudanças na legislação brasileira que garantam a ampliação do número de ACS por equipe. Outra demanda é a destinação de carga horária para treinamento e capacitação desses recursos humanos para a atuação junto à população idosa, com destaque para os dependentes.

Em decorrência do envelhecimento da população, que amplia o número de idosos mais velhos, os ACS têm grande importância e precisam estar cada vez mais preparados para o atendimento das necessidades básicas e das demandas assistenciais a esta clientela.

Esta investigação também contribui no campo da Saúde Coletiva no sentido de preencher lacunas do conhecimento na literatura científica nacional e internacional quanto ao objeto de estudo analisado, dada a carência de publicações sobre a atuação do ACS na interface do cuidado ao idoso dependente, o que confere caráter de originalidade e ineditismo ao texto.

Como limitação, verificou-se que, embora tenha emergido dos discursos a ideia de que o ACS é considerado importante na linha de cuidado ao idoso na APS, os questionamentos das entrevistas não abordaram este tema específico, o que pode ter suprimido a opinião de alguns participantes sobre esta temática. De outra parte, mesmo sem esse aspecto, muitos profissionais e gestores entrevistados, da maioria dos municípios, emitiram opiniões relevantes sobre a atuação do ACS no contexto da saúde do idoso.

\section{Colaboradores}

CCP Brasil, RM Silva, IC Bezerra, LJES Vieira, MLF Figueiredo, FRVF Castro, FFSN Queiroz e MRTF Capelo declaram que participaram da elaboração do manuscrito e que assumem publicamente a responsabilidade pelo seu conteúdo. 


\section{Referências}

1. Silva TL, Soares AN, Lacerda GA, Mesquita JFO, Silveira DC. Política Nacional de Atenção Básica 2017: implicações no trabalho do Agente Comunitário de Saúde. Saúde Debate 2020; 44(124):58-69.

2. Brasil. Ministério da Saúde (MS). Portaria no 2.436, de 21 de setembro de 2017. Aprova a Política Nacional de Atenção Básica, estabelecendo a revisão de diretrizes para a organização da Atenção Básica, no âmbito do Sistema Único de Saúde (SUS). Diário Oficial da União 2017; 22 set.

3. Brasil. Ministério da Saúde (MS). Agente Comunitário de Saúde [site da Internet]. Brasília: MS; 2020 [acessado 2020 Jun 10]. Disponível em: https://www.saude. gov.br/acoes-e-programas/saude-da-familia/agente-comunitario-de-saude

4. Butcher N, Sittherb A, Velavanc J, Johnd E, Thomase MC, Grills N. Evaluation of community health worker training course effectiveness in India. Christ $J$ Glob Health 2016; 3(2):18-26.

5. Giovanella L, Almeida PF. Atenção primária integral e sistemas segmentados de saúde na América do Sul. Cad Saude Publica 2017; 33(Supl. 2):e00118816.

6. Faria CCMV, Paiva CHA. O trabalho do agente comunitário de saúde e as diferenças sociais no território. Trab Educ Saúde [periódico na Internet] 2020 [acessado 2020 Abr 24]; 18(Supl. 1):e0025183. Disponível em: https://www.scielo.br/pdf/tes/ v18s1/1678-1007-tes-18-s1-e0025183.pdf

7. Guanaes-Lorenzi C, Pinheiro RL. A (des)valorização do agente comunitário de saúde na Estratégia Saúde da Família. Cienc Saude Colet 2016; 21(8):2537-2546.

8. Komaromy M, Ceballos V, Zurawski A, Bodenheimer T, Thom DH, Arora S. Extension for Community Healthcare Outcomes (ECHO):a new model for community health worker training and support. J Public Health Policy 2018; 39(2):203-216.

9. Torres S, Labonté R, Spitzer DL, Andrew C, Amaratunga $\mathrm{C}$. Improving health equity: the promising role of community health workers in Canada. Healthc Policy 2014; 10(1):73-85.

10. Prentiss T, Tamler I, Plum A, Parke D, Zervos J, Tanniru M, Sears MH, White M, Wisdom K, Kaljee L. Community health workers as innovators: methods and results from a tele-education pilot for community health workers in Detroit, Michigan. In: Anais do $50^{\circ}$ Hawaii International Conference on System Sciences (HICSS); 2017; Honolulu. p. 3273-3282.

11. Loureiro LH, Diogo MA, Mendes TB, Machado TBFV, Marcellini PS, Tonini T. O trabalho e a formação do agente comunitário de saúde. Rev Práxis 2017; 9(17):103-111.

12. Melo MB, Quintão AF, Carmo RF. O Programa de Qualificação e Desenvolvimento do Agente Comunitário de Saúde na perspectiva dos diversos sujeitos envolvidos na atenção primária em saúde. Saúde Soc 2015; 24(1):86-99.

13. Braga GMAM, Mafra SCT, Silva EP, Gomes AP, Melo MSS. Percepção do trabalho do agente comunitário de saúde pelos usuários atendidos nas unidades básicas de saúde da família de Viçosa, MG: tarefas realizadas e normas prescritas. Oikos 2016; 27(1):79-95.-
14. Andrade IA, Guimarães CP, Martins LC, Moreira JC. Capacitação em saúde do idoso para os agentes comunitários de saúde da UBS Citrolândia em Betim-MG: relato de uma prática intervencionista. Sinapse Múltipla 2017; 6(2):267-272.

15. Habermas J. Dialética e Hermenêutica. Porto Alegre: LPM; 1987.

16. Gadamer HG. Verdade e método: traços fundamentais de uma hermenêutica filosófica. Petrópolis: Vozes; 1999.

17. Minayo MCS. Hermenêutica dialética como caminho do pensamento social próprio. In: Minayo MCS, Deslandes SF, organizadores. Caminhos do pensamento: epistemologia e método. Rio de Janeiro: Fiocruz; 2013. p. 83-107.

18. Gomes MHA, Silveira C. Sobre o uso de métodos qualitativos em Saúde Coletiva, ou a falta que faz uma teoria. Rev Saude Publica 2012; 46(1):160-165.

19. Taquette SR. Análise de dados de pesquisa qualitativa em saúde. In: Atas do V Congresso Ibero-Americano em Investigação Qualitativa - CIAIQ; 2016; Porto, Portugal. p. 524-533.

20. Nascimento LCN, Souza TV, Oliveira ICS, Moraes JRMM, Aguiar RCB, Silva LF. Saturação teórica em pesquisa qualitativa: relato de experiência na entrevista com escolares. Rev Bras Enferm 2018; 71(1):228233.

21. Brasil. Ministério da Saúde (MS). Diretrizes para capacitação de agentes comunitários de saúde em linhas de cuidado. Brasília: MS; 2016.

22. Assis AS, Castro-Silva CR. Agente Comunitário de Saúde e o idoso: visita domiciliar e práticas de cuidado. Physis [periódico na Internet]. 2018 [acessado 2018 Out 23]; 28(3):0103-7331. Disponível em: https://www.scielo.br/pdf/physis/v28n3/0103-7331physis-28-03-e280308.pdf

23. Costa SM, Araújo FF, Martins LV, Nobre LLR, Araújo FM, Rodrigues CAQ. Agente Comunitário de Saúde: elemento nuclear das ações em saúde. Cien Saude Colet 2013; 18(7):2147-2156.

24. Muniz EA, Freitas CASL, Oliveira EM, Lacerda MR. Atenção domiciliar ao idoso na estratégia saúde da família: perspectiva sobre a organização do cuidado. Rev Enferm UFPE [periódico na Internet]. 2017 Jan [acessado 2020 Maio 23]; 11(Supl.1):296-302. Disponível em: https://periodicos.ufpe.br/revistas/revistaenfermagem/article/viewFile/11908/14391

25. Morosini MV. Educação e trabalho em disputa no SUS: a política de formação dos agentes comunitários de saúde. Rio de Janeiro: EPSJV; 2010.

26. Filgueiras AS, Silva ALA. Agente Comunitário de Saúde: um novo ator no cenário da saúde do Brasil. Physis [periódico na Internet]. 2011 [acessado 2020 Maio 23]; 21(3):899-916. Disponível em: https:// www.scielo.br/pdf/physis/v21n3/08.pdf

27. Magalhães KA, Giacomin KC, Santos WJ, Firmo JO. A visita domiciliária do agente comunitário de saúde a famílias com idosos frágeis. Cien Saude Coletiva [periódico na Internet]. 2015 [acessado 2020 Jan 23]; 20(12):3787-3796. Disponível em: https://www.scielosp.org/article/csc/2015.v20n12/3787-3796 
28. Litzelman DK, Inui TS, Griffin WJ, Perkins A, Cottingham AH, Schmitt-Wendholt KM, Ivy SS. Griffin W. Impact of community health workers on elderly patients' advance care planning and health care utilization: moving the dial. Med Care 2017; 55(4):319326.

29. Tulenko K, Møgedal S, Afzal MM, Frymus D, Oshin A, Pate M, Quain E, Pinel A, Wynd S, Zodpey S . Community health workers for universal health-care coverage: from fragmentation to synergy. Bull World Health Organization 2013; 91:847-852.

30. Butcher N, Grills N. Impacts of community lay-leader health worker training \& practice (Uttarakhand, India). Christ J Glob Health 2017; 4(2):43-52.

31. Javanparast S, Windle A, Freeman T, Baum F. Community health worker programs to improve healthcare access and equity: are they only relevant to lowand middle-income countries? Int J Health Policy Manag 2018; 7(10):943-954.

32. Brito LP. O agente comunitário em saúde: um cuidador ou um apoiador institucional? [dissertação]. Brasília: Universidade de Brasília; 2015.

33. Silva TL, Soares AN, Lacerda GA, Mesquita JFO, Silveira DC. Política nacional de atenção básica 2017: implicações no trabalho de agente comunitário de saúde. Saúde Debate [periódico na Internet]. 2020 [acessado 2020 Jan 23]; 44(124):58-69. Disponível em: https://www.scielosp.org/article/sdeb/2020. v44n124/58-69/pt/

34. Minayo MCS. O imperativo de cuidar da pessoa idosa dependente. Cien Saude Colet 2019; 24(1):247-252.

35. Instituto de Mayores y Servicios Sociales (IMSERMO). Libro Blanco de Atención a las personas en situación de dependencia en España. Madrid: IMSERMO; 2004 [acessado 2018 Jul 12]. Disponível em: https:// www.imserso.es/InterPresent2/groups/imserso/documents/binario/libroblanco.pdf

36. Fortes KMGS, Moura MEB, Nunes BMVT, Landim CAP, Lago EC. Formação do Agente Comunitário de Saúde da família na atenção ao idoso. Rev Enferm UFPE 2016; 10(Supl. 1):211-217.

37. Silva DM, Silva MAC, Oliveira DS, Alves M. Cotidiano de agentes comunitários de saúde com idosos segundo o referencial de certeau. Cogitare Enferm 2017; 22(4):e50436.

38. Medeiros KKAS, Pinto Júnior EP, Bousquat A, Medina MG. O desafio da integralidade no cuidado ao idoso, no âmbito da Atenção Primária à Saúde. Saúde Debate 2017; 41(3):288-295.
39. Rahmawati R, Bajorek B. A community health worker-based program for elderly people with hypertension in Indonesia: a qualitative study, 2013. Prev Chronic Dis 2015; 12:e175.

40. Heimann LS, Ibanhes LC, Boaretto RC, Castro IEN, Telesi Júnior E, Cortizo CT, Fausto MCR, Nascimento VBN, Kayano J. Atenção primária em saúde: um estudo multidimensional sobre os desafios e potencialidades na região metropolitana de São Paulo (SP, Brasil). Cienc Saude Colet [periódico na Internet]. 2011[acessado 2020 Jan 23]; 16(6):2877-2887. Disponível em: https://www.scielo.br/pdf/csc/v16n6/25. pdf

41. Brasil. Ministério da Saúde (MS). Política Nacional de Educação Permanente em Saúde: o que se tem produzido para o seu fortalecimento? Brasília: MS; 2018.

42. Peixoto LS, Gonçalves LC, Costa TD, Tavares CMM, Cavalcante ACD, Cortez EA. Educação permanente, continuada e em serviço: desvendando seus conceitos. Enferm Global 2013; 12(29):324-340.

43. Melo EA, Mendonça MHM, Oliveira JR, Andrade GCL. Mudanças na Política Nacional de Atenção Básica: entre retrocessos e desafios. Saúde Debate [periódico na Internet]. 2018 Set [acessado 2020 Maio 23]; 42(1):38-51. Disponível em: https://www.scielo.br/ pdf/sdeb/v42nspe1/0103-1104-sdeb-42-spe01-0038. pdf

44. Perry HB, Zulliger R, Rogers MM. Community health workers in low-, middle-, and high-income countries: an overview of their history, recent evolution, and current effectiveness. Annu Rev Public Health 2014; 35:399-421.

Artigo apresentado em 22/07/2020

Aprovado em 21/08/2020

Versão final apresentada em 23/08/2020

Editores chefes: Maria Cecília de Souza Minayo, Romeu Gomes, Antônio Augusto Moura da Silva. Editora Associada da área de Saúde do Idoso: Joselia Oliveira Araújo Firmo 